earthquakes as they happen - which is constantly. This initiative is called CUBE (Caltech-USGS Broadcast of Epicenters).

Because of initiatives such as SCEC, "my guess is that 50 years from now, earthquakes will be much less of a problem for human life", says David Jackson, professor of geophysics at the University of California, Los Angeles (UCLA). There will always be property damage, and part of SCEC's job is to advise engineers on what they can get away with, rather than devoting enormous sums to proof against really big earthquakes that may not happen in the building's projected lifetime. Nevertheless, money is a problem: "the cost of seismic upgrading right here on the UCLA campus during the 1990 s is something very close to $\$ 100$ million", says Jackson. Such figures make the $\$ 3$ million a year for the SCEC a price worth paying.

SLAC

\section{Ringing the changes}

MY directions to SLAC (the Stanford Linear Accelerator Center) were faultless, and I arrived an hour early. Twenty billion years earlier, though, the infant Universe had not the benefits of SLAC's public relations department, and somewhere along the line took a wrong turning: the result was a slight excess of regular matter over antimatter. The rest is history, and were it not for that excess, the Universe would be free of matter of any kind, and we would not be here to talk about it. It is embarrassing, therefore, that the Standard Model with which physicists like to explain everything cannot really explain the source of this initial discrepancy, called CP (charge-conjugation-times-parity) violation.

For something so fundamental, the only generally accepted experimental evidence for $\mathrm{CP}$ violation comes from the decay of a particle called the neutral $\mathrm{K}$ meson. Another design concerns another meson, the B meson. There are reasons for thinking that, every now and then, B and anti-B mesons decay in slightly different ways characteristic of $\mathrm{CP}$ violation. The problem is that such discrepancies would be tiny, the events rare, and that B mesons (discovered in 1977) are exotic to start with. A way to produce a lot of them would be to splurge beams of positrons and electrons together. If the beams were really intense, just now and then one of the results would be a particle called an upsilon, which decays into a B meson and its antiparticle, both of which would decay into a collection of other neutral and charged particles.
Close examination of the constituents in each case might reveal $\mathrm{CP}$ violation and, hopefully, somthing about its nature.

SLAC is ideally placed for such an experiment. It is a world leader in electron-positron beam technology, and could perform it with a (relatively) inexpensive add-on to an existing electronpositron storage ring. But there is not enough money to go round. In itself, that is not news, and the philosophical would heave a sigh and say that as the problem has been with us since the beginning of the Universe, a few more years will hardly matter. But history has conspired that SLAC as a whole will be in a perilous position were it not to secure funding for a B-factory - and soon.

It was not always thus. SLAC's Director, Burton Richter, came to Stanford in 1956 to do physics with electron beams, the constant theme of his career: it was thus only natural for him to become involved in Stanford's brand new linear electron accelerator from its inception. In 1968 , just two years after construction was complete, researchers at SLAC found the first evidence for quarks. In 1971, work started on SPEAR, a 8-GeV electron-positron ring. In what became known as the 'November revolution' in 1974, SLAC researchers working on SPEAR found the $J / \psi$ particle, indirect evidence for the charmed quark, work that led to a Nobel for Richter (shared with Sam Ting from MIT, who with his colleagues found the particle at Brookhaven). That Nobel year, 1976, SLAC researchers at SPEAR did it again, with the discovery of the $\tau$ lepton.

Then things started to unravel. A whole raft of information on the structure of matter has been prized from the 36-GeV PEP (positron-electron project) ring, started in 1976 and completed in 1980 , but the top quark forever remained just out of reach. Since 1991, PEP has remained quiet, and may stay that way unless SLAC gets the go-ahead to upgrade it into a B-meson factory. A year earlier, 1990, the venerable SPEAR was converted to a synchrotron radiation facility that is being used for a myriad experiments - but not particle physics.

Then came the Stanford Linear Collider (SLC), the electron-positron collider that raced against the LEP machine at CERN to produce large numbers of $\mathbf{Z}^{0}$ bosons and thus constrain the number of particle 'families' in nature to three. Even though SLC can do things that LEP can't, such as polarizing its beams of electrons to show that $\mathrm{Z}^{0}$ s are produced, preferentially, in 'left-handed' beams, it has never matched LEP for sheer numbers of particles.

Posterity will hail the SLC as the pioneer of a new age of linear colliders. "Everyone agrees that storage-ring technology has finished with LEP," says

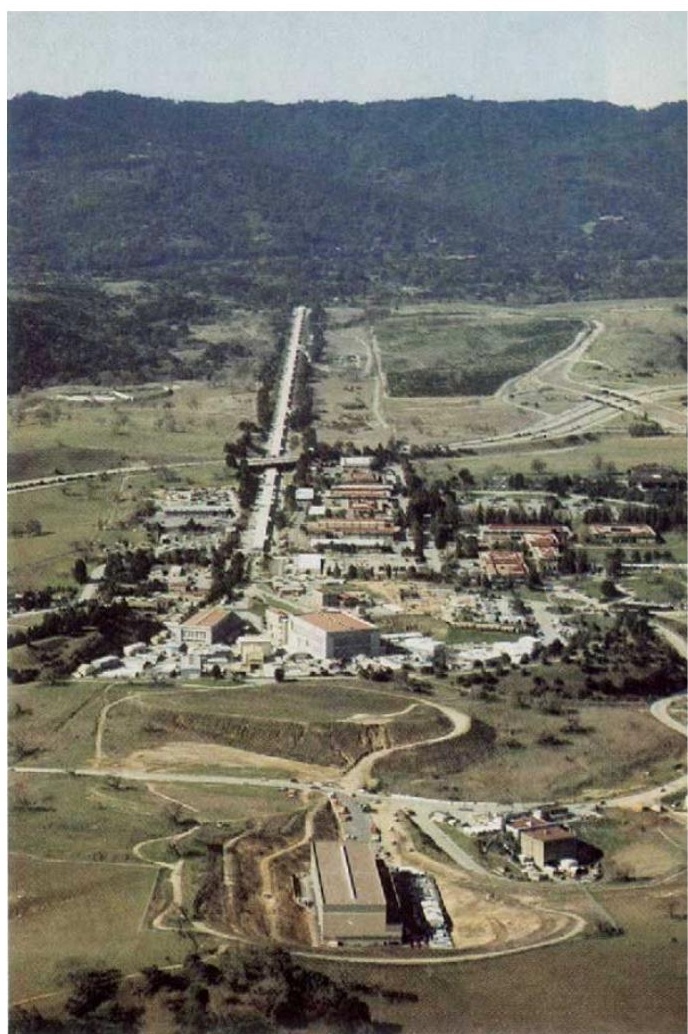

SLAC: still with a future.

Richter. "The only way to go to higher energies with the electron-positron system is with the linear collider. And there will be, some time in the early 2000 s, a linear collider at high energy - and this one is going to be an international collaboration". Indeed plans for a more powerful linear collider are being drawn up, involving researchers from the US, Europe, Japan and Russia.

Such an ambitious project seems some years from fruition, and in the meantime SLAC must get by on R\&D and synchrotron experiments. There are worries that without the immediate charge of cuttingedge particle physics offered by the B factory, SLAC is left exposed to cuts. A plan to streamline SLAC's operation so that the B-factory could be fitted into the operating budget, provided that the rest of SLAC's operations remained funded, met with the response that were instituted, the saving could be devoted to work at other DoE laboratories - and SLAC would have to wait for its B-factory, possibly until 1995. B-factories elsewhere would have had the chance to catch up on SLAC's lead.

But prospects at present seem slightly brighter than what was always a worstcase scenario. The end of the Cold War has allowed the DoE to be more flexible in its budgeting, and provision for the Bfactory appeared in budget plans drawn up by the Bush administration. Whether the Clinton administration adopts the Bfactory remains to be seen, but opinion within SLAC seems optimistic that funding will be appropriated. 